\title{
Spectral reflectance of solar light from dirty snow: a simple theoretical model and its validation
}

\author{
A. Kokhanovsky \\ Institute of Environmental Physics, University of Bremen, O. Hahn Allee 1, 28334 Bremen, Germany \\ Correspondence to: A. Kokhanovsky (alexk@iup.physik.uni-bremen.de) \\ Received: 16 January 2013 - Published in The Cryosphere Discuss.: 18 February 2013 \\ Revised: 4 July 2013 - Accepted: 17 July 2013 - Published: 20 August 2013
}

\begin{abstract}
A simple analytical equation for the snow albedo as the function of snow grain size, soot concentration, and soot mass absorption coefficient is presented. This simple equation can be used in climate models to assess the influence of snow pollution on snow albedo. It is shown that the squared logarithm of the albedo (in the visible) is directly proportional to the soot concentration. A new method of the determination of the soot mass absorption coefficient in snow is proposed. The equations derived are applied to a dusty snow layer as well.
\end{abstract}

\section{Introduction}

Snow cover and its spectral albedo are important factors influencing climate (Hansen and Nazarenko, 2004). Therefore, it is of importance to understand in what way various snow pollutants (e.g., soot, dust, algae) influence snow albedo and, therefore, duration of snow cover. Corresponding studies have been performed by Warren and Wiscombe (1980), Painter et al. (2001), Dozier and Painter (2004), Takeuchi et al. (2006), Aoki et al. (2007), Hadley and Kirchstetter (2012), and Warren (2013) among others. It was found that pollutants considerably modify snow spectral reflectance in the visible and near infrared (up to approximately the wavelength $\lambda=1 \mu \mathrm{m}$ ). This is due to the fact that pure snow belongs to the class of weakly absorbing and strongly multiply light scattering turbid media in the visible and near IR. Therefore, even small amounts of pollutants have a drastic influence on spectral reflectance (especially in the visible). This is due to the enhancement of light absorption by pollutants in response to their placement in a multiple light scattering environment. For instance, Hadley and
Kirchstetter (2012) found $20 \%$ decrease in snow albedo (at $\lambda=0.412 \mu \mathrm{m})$ for the soot concentration $c=1.68 \mu \mathrm{gg}^{-1}$ at the effective radius of snow grains of $55 \mu \mathrm{m}$ and nadir illumination of the snow layer; $10 \%$ reduction in albedo was observed at $c=0.45 \mu \mathrm{gg}^{-1}$ and $5 \%$ at $c=0.11 \mu \mathrm{gg}^{-1}$. Pure snow spectral albedo is close to unity and almost spectrally neutral in the visible. With addition of soot, the albedo becomes less spectrally neutral (as compared to pristine snow) with slight decrease in amount of reflected light towards $400 \mathrm{~nm}$. The most pronounced decrease towards UV is observed, if dust is added to the snow layer. Painter et al. (2007) discovered that the reflectance of snow at $412 \mathrm{~nm}$ is reduced by $50 \%$ when dust with $c=0.37 \mathrm{mg} \mathrm{g}^{-1}$ was deposited on a snow layer. This is due to the fact that the imaginary part of the dust refractive index $\chi$ increases towards $\mathrm{UV}$, which is not the case for soot. The reflectance is also reduced due to the presence of algae (e.g., light absorption at $0.4-0.6 \mu \mathrm{m}$ due to carotenoids and chlorophylls of snow algae). Takeuchi et al. (2006) reported $25 \%$ decrease in snow reflectance at $412 \mathrm{~nm}$ due to the presence of red snow algae (Chlamydomonas cf. nivalis).

The task of this paper is to present and validate a new model of dirty snow reflectance. It is based on the asymptotic radiative transfer theory valid for weakly absorbing optically thick media. Multiple light scattering effects in dirty snow are fully taken into account. In particular, the approximate analytical equations for the global (e.g., albedo) and local (e.g., extinction and absorption coefficients) snow optical properties are presented. This enables the derivation of direct analytical relationships between the snow reflectance characteristics and snow microstructure parameters such as the snow grain size and concentration of various pollutants (soot, dust, algae). The case of a semi-infinite snow layer is 
considered in detail. However, the model can be easily extended to the case of a finite snow layer as well as for the cases when the solar light transmittance through a snowpack is measured (Kokhanovsky, 2006). The model is presented in Sect. 2. Section 3 is aimed at the validation of the model using the experimental measurements of Painter et al. (2007), Brandt et al. (2011), and Hadley and Kirchstetter (2012).

\section{Exponential approximation}

The snow albedo $A$ can be represented as series with respect to the single scattering albedo $\omega_{0}=1-\beta$, where $\beta=k_{\mathrm{abs}} / k_{\mathrm{ext}} \quad\left(k_{\mathrm{abs}(\mathrm{ext})}\right.$ is snow absorption (extinction) coefficient). Namely, it follows:

$A=\sum_{m=1}^{\infty} a_{m}(1-\beta)^{m}$

with the normalization condition

$\sum_{m=1}^{\infty} a_{m}=1$

due to the energy conservation law ( $A=1$ in the absence of absorption processes). Here $m$ can be interpreted as a scattering event number. The expansion coefficients $a_{m}$ represent the strength of the contribution of the $m$-order scattering event to the snow albedo. Let us use the following expansion:

$(1-\beta)^{m}=\sum_{j=0}^{m}(-1)^{j} D_{m j} \beta^{j}$,

where $D_{m j}=m ! / j !(m-j) !$. The substitution of Eq. (3) in Eq. (1) gives

$A=1-\beta\langle m\rangle+\frac{\beta^{2}\left\langle m^{2}\right\rangle}{2}-\frac{\beta^{3}\left\langle m^{3}\right\rangle}{6} \approx\langle\exp (-\beta m)\rangle$,

where

$$
\begin{aligned}
& \left\langle m^{k}\right\rangle=\sum_{m=1}^{\infty} a_{m} m^{k}, \\
& \langle\exp (-\beta m)\rangle=\sum_{m=1}^{\infty} a_{m} \exp (-\beta m)
\end{aligned}
$$

and we assumed that $m(m-1) \approx m^{2}, m(m-1)(m-2) \approx$ $m^{3}, \ldots$ in the derivation of Eq. (4), which is a valid assumption due to a large number of scattering events $m$ in weakly absorbing snow. The summation in Eq. (6) can be performed analytically transferring to the continuous basis with respect to the parameter $m$ and using the results of the random walk theory (Chandrasekhar, 1943):

$a(m)=\sqrt{p / \pi} m^{-3 / 2} \exp (-p / m)$, where the parameter $p$ depends on the process under study. The integral

$A=\int_{0}^{\infty} a(m) \exp (-\beta m) \mathrm{d} m$

can be evaluated analytically (Kokhanovsky, 2002):

$A=\exp (-2 \sqrt{p \beta})$.

The value of $p$ is found by taking into account that it follows from the radiative transfer theory as $\beta \rightarrow 0$ (Kokhanovsky, 2006):

$A=1-4 \sqrt{\frac{\beta}{3(1-g)}}$,

where $g$ is the average cosine of light scattering angle inside the snow layer. Therefore, it follows:

$p=\frac{4}{3(1-g)}$

and

$A=\exp (-\alpha)$

where

$\alpha=4 \sqrt{\frac{\beta}{3(1-g)}}$.

Equation (12) can be also used to find the plane albedo $r\left(\mu_{0}\right)$ and reflection function $R\left(\mu_{0}, \mu, \phi\right)$ (Kokhanovsky, 2006):

$r\left(\mu_{0}\right)=A^{K\left(\mu_{0}\right)}$,

$R\left(\mu_{0}, \mu, \phi\right)=R_{0}\left(\mu, \mu_{0}, \phi\right) A^{v}$,

where $K\left(\mu_{0}\right)=\frac{3}{7}\left(1+2 \mu_{0}\right), v=K\left(\mu_{0}\right) K(\mu) R_{0}^{-1}\left(\mu_{0}, \mu, \phi\right)$ $\mu_{0}$ is the cosine of the solar zenith angle, $\mu$ is the cosine of the observation zenith angle, and $\phi$ is the relative azimuthal angle. Simple parameterization for the function $R_{0}\left(\mu, \mu_{0}, \phi\right) \equiv$ $R\left(\mu, \mu_{0}, \phi, \omega_{0}=1\right)$ was presented by Kokhanovsky (2006). This function depends on the viewing geometry and the shape of grains. Its dependence on the size of ice grains can be ignored. The snow bidirectional reflection distribution function (BRDF) is proportional to the reflection function $R\left(\mu_{0}, \mu, \phi\right)$ : $\operatorname{BRDF}=R\left(\mu_{0}, \mu, \phi\right) / \pi$.

The next step in our derivation is to relate the parameter $\alpha$ (or $\beta, g$, see Eq. 13) with the size of snow grains and parameters of impurities (concentration, size, etc.). We will assume that snow consists of ice grains and other scatterers (e.g., soot, dust, algae, etc.) suspended in air. The dense media effects are ignored. Then the snow extinction coefficient $k_{\text {ext }}$, absorption coefficient $k_{\text {abs }}$, scattering coefficient 
$k_{\text {sca }}=k_{\text {ext }}-k_{\text {abs }}$, phase function $p(\theta)$, and average cosine of scattering angle $g$ can be presented as

$$
\begin{aligned}
k_{\mathrm{ext}} & =k_{\mathrm{ext}}^{\mathrm{ice}}+\sum_{j=1}^{M} k_{\mathrm{ext}, j} \\
k_{\mathrm{abs}} & =k_{\mathrm{abs}}^{\mathrm{ice}}+\sum_{j=1}^{M} k_{\mathrm{abs}, j}, \\
k_{\mathrm{sca}} & =k_{\mathrm{sca}}^{\mathrm{ice}}+\sum_{j=1}^{M} k_{\mathrm{sca}, j}, \\
p(\theta) & =\frac{k_{\mathrm{sca}}^{\mathrm{ice}} p^{\mathrm{ice}}(\theta)+\sum_{j=1}^{M} k_{\mathrm{sca}, j} p_{j}(\theta)}{k_{\mathrm{sca}}^{\mathrm{ice}}+\sum_{j=1}^{M} k_{\mathrm{sca}, j}}, \\
g & =\frac{k_{\mathrm{sca}}^{\text {ice }} g^{\mathrm{ice}}+\sum_{j=1}^{M} k_{\mathrm{sca}, j} g_{j}}{k_{\mathrm{sca}}^{\text {ice }}+\sum_{j=1}^{M} k_{\mathrm{sca}, j}},
\end{aligned}
$$

where "ice" means that the parameter corresponds to the pure snow (no impurities, only ice phase) and the account for $M$ impurities is given in the second terms of corresponding expressions. The probability of photon absorption is calculated as $\beta=k_{\mathrm{abs}} / k_{\mathrm{ext}}$ and the asymmetry parameter $g=\frac{1}{2} \int_{0}^{\pi} p(\theta) \sin \theta \cos \theta \mathrm{d} \theta$. All local optical characteristics given above can be computed using the Mie theory for spherical particles (Mie, 1908) or geometrical optics approaches (van de Hulst, 1981; Liou, 2002; Kokhanovsky, 2006) for particles of different shapes; also approximations, look-uptables, and parameterizations of results of exact calculations are possible. The selected approximations are discussed below.

The extinction and absorption coefficients of the pure ice can be presented as

$k_{\mathrm{ext}}^{\text {ice }}=N C_{\mathrm{ext}}, \quad k_{\mathrm{abs}}^{\mathrm{ice}}=N C_{\mathrm{abs}}$.

Here $C_{\text {ext }}$ and $C_{\text {abs }}$ are average extinction and absorption cross sections of snow grains and $N=c_{\mathrm{i}} / V$ is the number concentration of snow grains $\left(c_{\mathrm{i}}\right.$ is their volumetric concentration and $V$ is the average volume of grains). It follows in the framework of the geometrical optics approximation that (Kokhanovsky, 2006)

$C_{\text {ext }}=2 \Sigma, \quad C_{\mathrm{abs}}=\xi \gamma V$

for large (as compared to the wavelength) randomly oriented ice grains of the arbitrary shape. The expression for $C_{\text {abs }}$ presented here is valid in the case of weakly absorbing large particles such as ice grains in the visible and near IR regions of the electromagnetic spectrum (Kokhanovsky and Zege, 2004). Here $\Sigma$ is the average projection area of the particles ( $\Sigma=S / 4$ for convex particles, where $S$ is the average surface area of the particles) and $\gamma=\frac{4 \pi \chi_{\mathrm{i}}(\lambda)}{\lambda}, \chi_{\mathrm{i}}$ is the imaginary part of the ice refractive index at the wavelength $\lambda$. The parameter $\xi$ depends on the shape of particles (being close to 1.28 for ice spheres, Kokhanovsky and Zege, 2004) and the real part of the ice refractive index $n$. This parameter characterizes the efficiency of absorption (EA) by a particle of a given shape and refractive index and equals the ratio $C_{\mathrm{abs}} / \gamma V$, which approaches 1 as $n \rightarrow 1$ independently on the shape of scatterers (van de Hulst, 1981). The dependence of EA on the size of particles can be ignored in the visible and near IR regions of the electromagnetic spectrum.

Let us introduce the effective grain size (EGS) as

$a_{\mathrm{ef}}=\frac{3 V}{4 \Sigma}$

The value of $a_{\mathrm{ef}}$ coincides with the radius of particles in the case of monodispersed spheres. One can then easily derive for the probability of photon absorption

$\beta=\sigma \gamma a_{\mathrm{ef}}$

where $\sigma=2 \xi / 3(\approx 0.85$ for spheres).

One derives for the parameter $\alpha$ (see Eq. 13) in the case of the pure (no pollutants) snow:

$\alpha=\sqrt{\gamma a_{\mathrm{ef}}} P$,

where $P=4 \sqrt{\sigma / 3(1-g)}$. The parameter $\alpha$ and, therefore, the grain size $a_{\text {ef }}$ can be derived from the snow reflectance measurements. However, for this the assumption on the value of $P$ must be made. The geometrical optics calculations for the spherical particles give $P=6.3$. The true value of $P$ depends on the type of snow (the shape of ice grains). It is difficult to calculate this quantity from the first principles due to the irregular shape of snow grains. Therefore, it is important to report both values $P$ and $a_{\mathrm{ef}}$ in outputs of corresponding retrieval algorithms. One can also introduce the optically characteristic length $a_{\mathrm{opt}}=P^{2} a_{\mathrm{ef}}$. This parameter can be directly derived from the reflectance measurements and less influenced by the assumptions on the shape of particles. The value of $a_{\text {opt }}$ can be also used to find the spectral snow albedo using the following simple approximation:

$A(\lambda)=\exp \left\{-\sqrt{\gamma(\lambda) a_{\mathrm{opt}}}\right\}$.

Let us consider the polluted snow now. We select soot as a single pollutant. Then we may assume that light scattering and extinction (but not absorption) in snow is dominated by ice grains. The equation for the parameter $\beta$ must be modified as

$\beta=\beta_{\text {ice }}+\beta_{\text {soot }}$

It follows (see Eq. 23):

$\beta_{\text {ice }}=\sigma \gamma a_{\text {ef }}$

Let us introduce the soot mass absorption coefficient $\varepsilon$ :

$k_{\mathrm{abs}}^{\mathrm{soot}}=\varepsilon \rho_{\mathrm{s}} c_{\mathrm{s}}$, 
where $\rho_{\mathrm{S}}$ is the soot density and $c_{\mathrm{s}}$ is the volumetric concentration of soot. Taking into account that $k_{\mathrm{ext}}=1.5 c_{\mathrm{i}} / a_{\mathrm{ef}}$ (Kokhanovsky, 2006), one derives

$\beta_{\mathrm{s}}=\frac{2 c_{\mathrm{s}}}{3 c_{\mathrm{i}}} \varepsilon \rho_{\mathrm{s}} a_{\mathrm{ef}}$.

We will assume that $\varepsilon \sim \lambda^{-1}$ (as for Rayleigh scatterers) and, therefore, $\varepsilon(\lambda)=\varepsilon\left(\lambda_{0}\right) \lambda_{0} / \lambda$. Taking into account the formulae given below, we arrive at the following equation:

$\alpha(\lambda)=\sqrt{\left(B_{\mathrm{i}} \chi_{\mathrm{i}}(\lambda)+B_{\mathrm{s}} c\right) x_{\mathrm{ef}}}$,

where

$B_{\mathrm{i}}=\frac{64 \xi}{9(1-g)}, \quad B_{\mathrm{s}}=\frac{16 \rho_{\mathrm{i}} \varepsilon\left(\lambda_{0}\right) \lambda_{0}}{9 \pi(1-g)}, \quad c=\frac{\rho_{\mathrm{s}} c_{\mathrm{s}}}{\rho_{\mathrm{i}} c_{\mathrm{i}}}, \quad x_{\mathrm{ef}}=\frac{2 \pi a_{\mathrm{ef}}}{\lambda}$,

and $\rho_{\mathrm{i}}=0.9167 \mathrm{~g} \mathrm{~cm}^{-3}$ is the ice density. The function $\alpha(\lambda)$ given in Eq. (30) can be used to find the spectral snow spherical albedo $A(\lambda)=\exp (-\alpha(\lambda))$, the plane albedo $r(\lambda)=$ $A(\lambda)^{K\left(\mu_{0}\right)}$ and also the snow BRDF as discussed above. The snow spectral absorptance $\Pi$ (for the diffuse illumination conditions) is calculated as

$\Pi=1-\exp \left[-\sqrt{\left(B_{\mathrm{i}} \chi_{\mathrm{i}}(\lambda)+B_{\mathrm{s}} c\right) x_{\mathrm{ef}}}\right]$.

All these characteristics are determined by just five main parameters: the size of particles $a_{\mathrm{ef}}$, the efficiency of light absorption by nonspherical ice grains $\xi$, the average cosine of scattering angle $g$, and the mass absorption coefficient and the concentration of a pollutant.

\section{Validation}

The comparison of calculations according to Eqs. (14) and (30) with experimental results for the plane albedo as presented by Hadley and Kirchstetter (2012) is given in Fig. 1. The excellent agreement of theory with the experiment is found assuming that $B_{\mathrm{i}}=77.02$ and $B_{\mathrm{s}}=22.15$. These parameters were derived using the minimization procedure at the largest concentration of pollutants at $a_{\mathrm{ef}}=55 \mu \mathrm{m}$ (see lower curve in Fig. 1a). Theoretical calculations for spherical particles such as those created in the experiment by Hadley and Kirchstetter (2012) result in (see Eq. 31 at $\xi=1.28, g=0.884$, Kokhanovsky and Zege, 2004) $B_{\mathrm{i}}=$ 78.47. It follows that theoretical and experimental values of $B_{\mathrm{i}}$ are in a good agreement. The theoretical value of $B_{\mathrm{S}}$ cannot be derived because the value of $\varepsilon\left(\lambda_{0}\right)$ for soot in snow was not determined experimentally. The experimental value of $B_{\mathrm{S}}=22.15$ is consistent with the assumption that $\varepsilon(0.435 \mu \mathrm{m})=9.27 \mathrm{~g} \mathrm{~cm}^{-2}$ (see Eq. 31), which is plausible for the laboratory experiment performed by Hadley and Kirchstetter (2012). Summing up, we conclude that simple equations presented here can indeed be used to simulate influence of snow pollution on albedo. Small deviations of experimental and theoretical results present in Fig. 1 could be a)

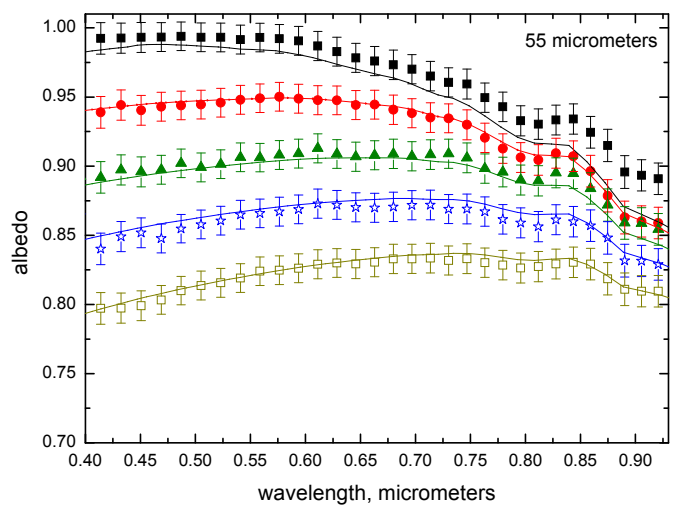

b)

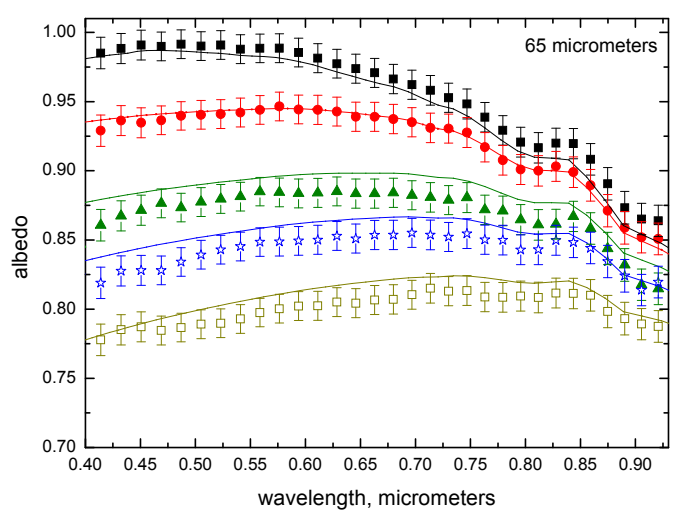

c)

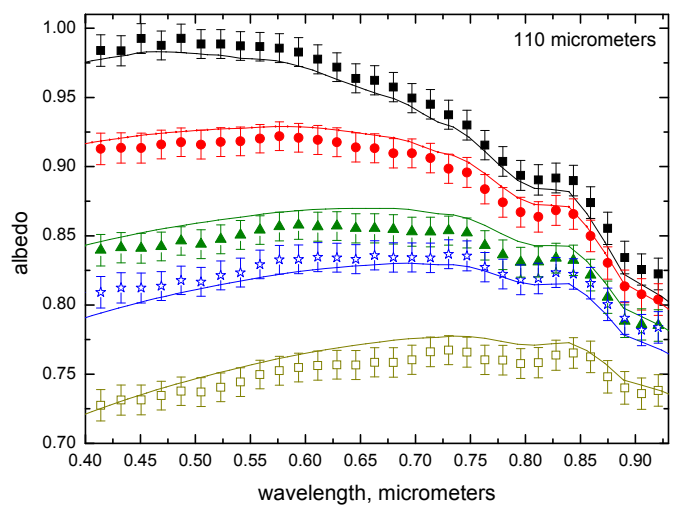

Fig. 1. The spectral dependence of snow plane albedo at the nadir illumination at various levels of snow pollution and effective radii - (a) 55, (b) 65, and (c) $110 \mu \mathrm{m}$. The results of calculations at $B_{\mathrm{i}}=77.02$ and $B_{\mathrm{S}}=22.15$ are given by lines. The dependence of $\chi_{\mathrm{i}}(\lambda)$ was taken from Warren and Brandt (2008). Symbols represent laboratory measurements performed by Hadley and Kirchstetter (2012) at concentrations $0,0.1,0.45,0.86$, and $1.68 \mu \mathrm{g} \mathrm{g}^{-1}$ (from top to bottom, respectively). 


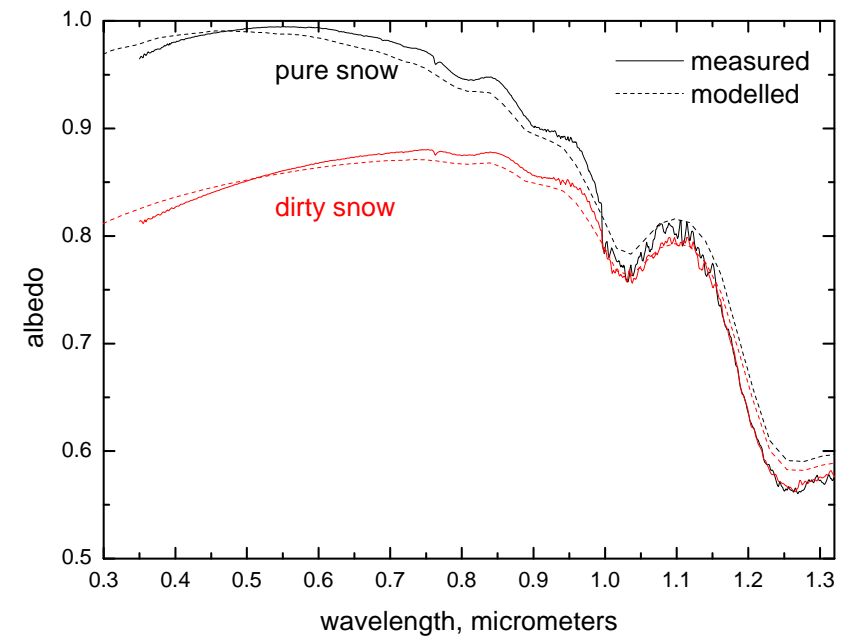

Fig. 2. The spectral dependence of snow spherical albedo at $c=$ $2.5 \mu \mathrm{gg}^{-1}$ and $a_{\mathrm{ef}}=55 \mu \mathrm{m}$. The results of calculations are given by broken lines. Solid lines represent experimental measurements for artificial snow composed of nearly spherical ice crystals (Brandt et al., 2011).

due to the variation of effective snow grain size for different levels of pollution.

The results of comparisons of calculations and measurements for yet another experiment with an artificial snow performed by Brandt et al. (2011) are presented in Fig. 2 for the case of spherical albedo. The exponential approximation for the spherical albedo

$A=\exp \left[-\sqrt{\left(B_{\mathrm{i}} \chi_{\mathrm{i}}(\lambda)+B_{\mathrm{s}} c\right) x_{\mathrm{ef}}}\right]$

was used in calculations (with $B_{\mathrm{i}}=78.47, B_{\mathrm{s}}=14.76$ ). The value of $B_{\mathrm{i}}$ for large spherical ice grains was calculated as described above. The dependence of $\chi_{\mathrm{i}}(\lambda)$ was taken from Warren and Brandt (2008). The value of $B_{\mathrm{S}}$ was derived from Eq. (31) assuming that $g=0.884$ (as for large spherical ice grains) and $\varepsilon(0.55 \mu \mathrm{m})=6 \mathrm{~m}^{2} \mathrm{~g}^{-1}$ as measured by Brand et al. (2011). It was assumed that $c=0.25 \mu \mathrm{gg}^{-1}$ and $a_{\mathrm{ef}}=55 \mu \mathrm{m}$ (as in the experiment). Therefore, no fitting procedure was applied. It follows that Eq. (33) can indeed be used to model both pure snow and dirty snow albedos. The small deviations of theoretical curve for pure snow from the measurements are due to the fact that (as mentioned by Brandt et al., 2011) the value of $a_{\text {ef }}$ was somewhat larger in case of pure snow. The disagreement of theory with the experiment around $0.4 \mu \mathrm{m}$ is most probably due to presence of the small amount of brown absorber in the water supply used to produce artificial snow (Brandt et al., 2011).

It should be emphasized that the value of $g$ in case of irregularly shaped ice grains is close to 0.75 and $\xi=1.84$ (Kokhanovsky and Zege, 2004). Therefore, it is expected that the value of $B_{\mathrm{S}}$ is somewhat smaller for snow composed of irregular snow grains as compared to the case of spherical scatterers (even for the same soot mass absorption coefficients).

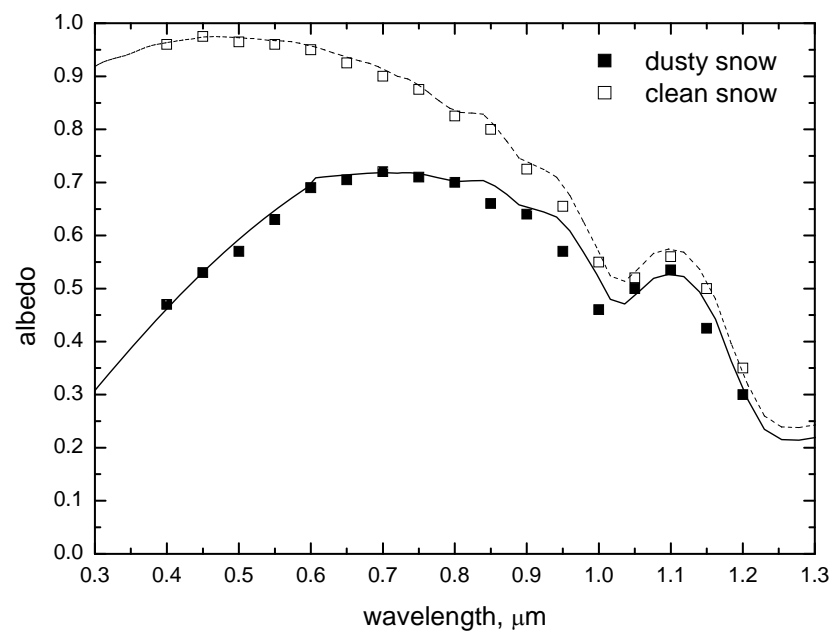

Fig. 3. The spectral dependence of clean and dusty snow spherical albedo at $c=0.22 \mathrm{mg} \mathrm{g}^{-1}$ and $a_{\mathrm{ef}}=400 \mu \mathrm{m}$ (for clean snow), $a_{\mathrm{ef}}=$ $450 \mu \mathrm{m}$ (for dusty snow). The results of calculations are given by lines. Symbols represent experimental measurements (Painter et al., 2007).

This will produce a smaller influence of soot on snow albedo as compared to that reported in this work and also in the experiments of Brandt et al. (2011) and Hadley and Kirchstetter (2012). Clearly, the albedo reduction is not solely the function of $c$ but also the size and shape of snow grains play a role (see Fig. 1).

Due to its high accuracy, Eq. (33) can be used for the determination of the soot mass absorption coefficient from the measurement of snow albedo at a given concentration of soot particles $c$. Namely, it follows from Eqs. (33) and (31):

$\varepsilon\left(\lambda_{0}\right)=\frac{9 \pi(1-g)}{16 \rho_{\mathrm{i}} \lambda_{0} c}\left\{\frac{\ln ^{2} A}{x_{\mathrm{ef}}}-B_{\mathrm{i}} \chi_{\mathrm{i}}\right\}$.

The derivations reported above have been performed for a specific case of soot as a single pollutant. However, the extension to other pollutants is easy. In particular, if light scattering occurs predominantly on ice grains (and not on snow impurities), Eq. (30) remains valid for other impurities (e.g., dust) except the value of $B_{\mathrm{s}}$ must be multiplied by the spectral function $f(\lambda)$, which accounts for the actual dependence of the impurity absorption coefficient on the wavelength. This function is equal to unity, if $\varepsilon \sim \lambda^{-1}$ as discussed above. For impurities, which both scatter and absorb light, Eqs. (16)-(19) must be used. We show the application of Eq. (33) to the interpretation of light reflectance from dusty snow in Fig. 3. Equation (33) can be re-written as

$A=\exp \left[-\sqrt{\left(B_{\mathrm{i}} \chi_{\mathrm{i}}(\lambda)+B_{\mathrm{d}} f c\right) x_{\mathrm{ef}}}\right]$,

where we introduced the spectral factor

$f=b \exp \left(-\lambda / \lambda_{0}\right)$. 
The constants $b=31.16$ and $\lambda_{0}=0.18 \mu \mathrm{m}$ were obtained using Eq. (36) for fitting the spectral dependence of the ratio of the imaginary part of dust refractive index taken from D'Almeida et al. (1991) (their Table 4.3) to its value at $0.7 \mu \mathrm{m}$. The value of $f$ was assumed to be equal 1.0 at the wavelengths larger than $0.6 \mu \mathrm{m}$. It was assumed that the effective radius of particles equal to $400 \mu \mathrm{m}$ for the clean snow and $450 \mu \mathrm{m}$ for the polluted snow. The value of $B_{\mathrm{d}}$ was set equal to 0.11 , which corresponds to the dust mass absorption coefficient (MAC) $0.046 \mathrm{~m}^{2} \mathrm{~g}^{-1}$ (Yasunari et al., 2011), if other settings are the same as for Fig. 2. The good fit can be obtained with the value of $c=0.22 \mathrm{mg} \mathrm{g}^{-1}$ (see Fig. 3). The value of $c$ depends on the assumption on the dust MAC, which was not measured for the experiment shown in Fig. 3. For multiple impurities in snow, Eq. (35) remains valid but the value of $B_{\mathrm{d}} f c$ must be substituted by $\sum_{j=1}^{M} B_{j} f_{j} c_{j}$, where $M$ is the number of impurities.

\section{Conclusions}

We have derived a simple exponential approximation, which can be used to relate the snow spherical and plane albedo (and also BRDF) to the soot and dust concentrations in snow. The derived analytical equations are very simple and can be used at snow field work, when the comprehensive radiative transfer codes are not available. The comparison of Fig. 1 of this work with corresponding figures given by Hadley and Kirchstetter (2012) and also Fig. 2 of this paper with Fig. 5 of Brandt et al. (2011) shows that the spectral albedo derived using Eq. (33) is very similar to that obtained using the exact solution of integro-differential radiative transfer equation in the framework of discrete-ordinates method (Stamnes et al., 1988). Eq. (33) was derived assuming the external mixture of soot and ice grains. In many cases soot in snow is better represented as a combination of external and internal (soot inside of ice grains) mixtures. To treat this case in a correct way, Eq. (31) for the value of $B_{\mathrm{s}}$ must be modified. In particular, this coefficient must be presented as a weighted sum of the value of $B_{\mathrm{S}}$ for the case of externally and internally mixed soot particles with the values of $B_{\mathrm{S}}$ being larger for the case of internally mixed soot particles (Kokhanovsky, 1989). We did not make an attempt to model the dirty snow as a combination of internally and externally mixed soot particles in snow grains for the experiments presented in this work because the concentration of internally mixed soot was not provided in corresponding papers. Therefore, the value of $B_{\mathrm{s}}$ given in Eq. (32) must be understood as an effective parameter, containing contributions both from internally and externally soot particles. The correctness of such an approach is confirmed by the accuracy of the resulted approximation as shown in Figs. 1-3. The proposed equations can be also used in global climate circulation models and to assess the impact of soot and other pollutants on the surface temperature, the duration of snow cover, the snowmelt, and the turbulent heat exchange between the surface and the lowest layer of atmosphere.

Acknowledgements. This work was supported by the BMBF Project CLIMSLIP and FP7 Project SIDARUS. It was conducted as part of the GCOM-C/SGLI Snow Project supported by Japan Aerospace Exploration Agency (JAXA). The author is grateful to R. E. Brandt and O. Hadley for providing experimental data in a tabular form.

Edited by: F. Dominé

\section{References}

Aoki, T., Hori, M., Motoyoshi, H., Tanikawa, T., Hachikubo, A., Sugiura, K., Yasunari, T. J., Storvold, R., Eide, H. A., Stamnes, K., Li, W., Nieke, J., Nakajima, Y., and Takahashi, F.: ADEOS-II/GLI snow/ice products - part II: validation results using GLI and MODIS data, Remote Sens. Environ., 111, 274-290, 2007.

Brandt, R. E., Warren, S. G., and Clarke, A. D.: A controlled snowmaking experiment testing the relation between black carbon content and reduction of snow albedo, J. Geophys. Res., 116, D08109, doi:10.1029/2010JD015330, 2011.

Chandrasekhar, S.: Stochastic problems in physics and astronomy, Rev. Mod. Phys., 15, 1-89, 1943.

D’Almeida, G. A., Koepke, P., and Shettle, E. P.: Atmospheric Aerosols. Global Climatology and Radiative Characteristics, A. Deepak Publishing, Hampton, 1991.

Dozier, J. and Painter, T. H.: Multispectral and hyperspectral remote sensing of alpine snow properties, Ann. Rev. Earth Planet. Sci., 32, 465-494, 2004.

Hadley, O. and Kirchstetter, W.: Black-carbon reduction of snow albedo, Nat. Clim. Change, 2, 437-440, 2012.

Hansen, J. and Nazarenko, L.: Soot climate forcing via snow and ice albedo, P. Natl. Acad. Sci. USA, 101, 423-428, 2004.

Kokhanovsky, A. A.: The geometrical optics approximation for the absorption cross section of a layered sphere, Atmospheric Optics, 2, 908-912, 1989.

Kokhanovsky, A. A.: Statistical properties of a photon gas in random media, Phys. Rev., E66, 037601, doi:10.1103/PhysRevE.66.037601, 2002.

Kokhanovsky, A. A.: Cloud Optics, Springer, Berlin, 2006.

Kokhanovsky, A. A. and Zege, E. P.: Scattering optics of snow, Appl. Optics, 43, 1589-1602, 2004.

Liou, K. N.: An Introduction to Atmospheric Radiation, Academic Press, NY, 2002.

Mie, G.: Beiträge zur Optik trüber Medien, speziell kolloidaler Metallösungen, Ann. Phys., 330, 377-445, 1908.

Painter T. H., Duval, B., Thomas, W. H., Mendez, M., Heintzelman, S., and Dozier, J.: Detection and quantification of snow algae with an airborne imaging spectrometer, Appl. Environ. Microbiol., 67, 5267-5272, 2001.

Painter, T. H., Barrett, A. P., Landry, C. C., Neff, J. C., Cassidy, M. P., Lawrence, C. R., McBride, K. E., and Farmer, G. L.: Impact of disturbed desert soils on duration of mountain snow cover, Geophys. Res. Lett., 34, L12502, doi:10.1029/2007GL030284, 2007. 
Stamnes, K., Tsay, S.-C., Wiscombe, W., and Jayaweera, K.: Numerically stable algorithm for discrete-ordinate-method radiative transfer in multiple scattering and layered media, Appl. Optics, 27, 2502-2509, doi:10.1364/AO.27.002502, 1988.

Takeuchi, N., Dial, R., Kohshima, S., Segawa, T., and Uetake, J.: Spatial distribution and abundance of red snow algae on the Harding Icefield, Alaska derived from a satellite image, Geophys. Res. Lett., 33, L21502, doi:10.1029/2006GL027819, 2006. van de Hulst, H. C.: Light Scattering by Small Particles, Dover, NY, 1981.

Warren, S. G.: Can be black carbon in snow be detected by remote sensing?, J. Geophys. Res., 118, 779-786, doi:10.1029/2012JD018476, 2013.
Warren, S. G. and Brandt, R. E.: Optical constants of ice from ultraviolet to the microwave: a revised compilation, J. Geophys. Res., 113, D14220, doi:10.1029/2007JD009744, 2008.

Warren, S. G. and Wiscombe, W.: A model of spectral albedo of snow, II: Snow containing atmospheric aerosols, J. Atmos. Sci., 37, 2734-2745, 1980.

Yasunari, T. J., Koster, R. D., Lau, K.-M., Aoki, T., Sud, Y. C., Yamazaki, T., Motoyoshi, H., and Kodama, Y.: Influence of dust and black carbon on the snow albedo in the NASA Goddard Earth Observing System version 5 land surface model, J. Geophys. Res., 116, D02210, doi:10.1029/2010JD014861, 2011. 